ИЗВЕСТИЯ АКАДЕМИИ НАУК ЭСТОНСКОП ССР. ТОМ 30 ФИЗИКА * МАТЕМАТИКА. 1981. № 1

\title{
ДИНАМИКА ОБРАЗОВАНИЯ НАЧАЛЬНОЙ ОБЛАСТИ ВКЛЮЧЕНИЯ В МОЩНЫХ ТИРИСТОРАХ
}

\author{
(Представил Н. Эпик)
}

Совершенствование мощных быстродействующих тиристоров идет по пути создания структур с многоразветвленными и протяженными управляющими электродами (УЭ). Эффективность работы таких тирнсторов, особенно в импульсных и высокочастотных режимах, в значительной степени определяется площадью начальной области включения (НОВ), характером ее формирования в процессе нарастания тока, а гакже параметрами цепи управления.

Динамика образования НОВ в тиристорах исследовалась в $\left[{ }^{1-4}\right]$. В $\left[{ }^{4}\right]$ было выведено эмпирическое выражение, связывающее площадь включения $S_{\mathrm{I}}=S(t)$ с конструкцией УЭ, со статическим отпирающим током управления $I_{G T}$ и параметрами внешнего сигнала управления. Однако разработка современных мощных тиристоров с разнообразными конструкциями УЭ, в том числе и регенеративных, вызывает необходимость в проведении дополнительных исследований динамнки формирования $\mathrm{HOB}$ в целях уточнения характера зависимости $S_{\text {н }}=$ $=S(t)$ в таких тиристорах.

\section{Аппаратура и методика исследования}

Блок-схема экспериментальной установки показана на рис. 1.

Инфракрасное излучение, выходящее из исследуемой тиристорной структуры (6), фокусируется объективом «Юпитер-3» на охлажденную парами жидкого азота катодную поверхность электронно-оптического преобразователя (ЭОП) типа У-72-1 (3), помещенного в герметичную камеру. В эту камеру вмонтировано «окно» из оптического стекла (a) с двойными стенками, между которыми создан вакуум, что обеспечивает высокую теплоизоляцию и проход регистрируемого излучения без ощутимых потерь. Питание на ӘОП поступает от высоковольтного источника (2).

В настоящей установке, в отличие от описанной в $\left[{ }^{1}\right]$, использован блок отсечения по времени (8), что позволяет путем варьирования продолжительности протекания нагрузочного тока на этапе его нарастания через исследуемую тиристорную структуру достигать временного разрешения.

Изображение НОВ на экране ЭОП фотографируется на пленку 


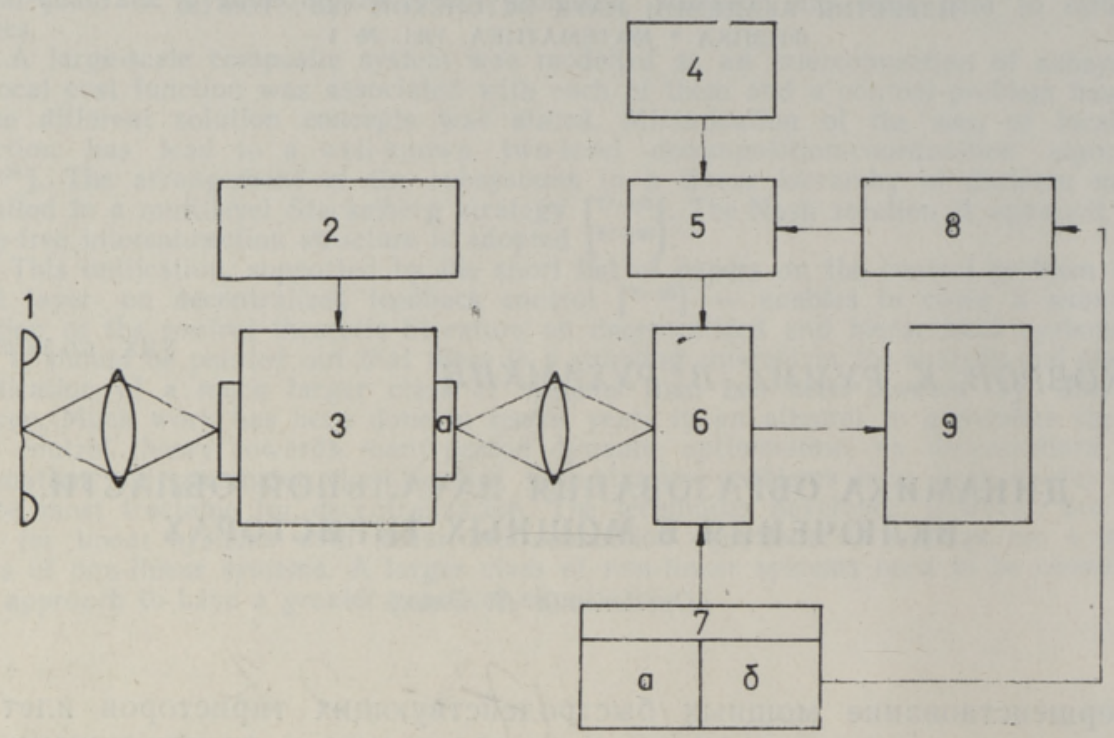

Рис. 1. Блок-схема экспернментальной установки: 1 - фотоаппарат, 2 - высоковольтный источник питания, 3 - камера ЭОП с вакуумиым «окном» $(a), 4-$ - зарядное устройство, 5 - RC-контур, 6 - исследуемая тиристорная структура, 7 - блок управления, включающиї источник постоянного тока (a) и генератор импульсного тока (б), 8 - блок отсечения по времени, 9 - осциллограф.

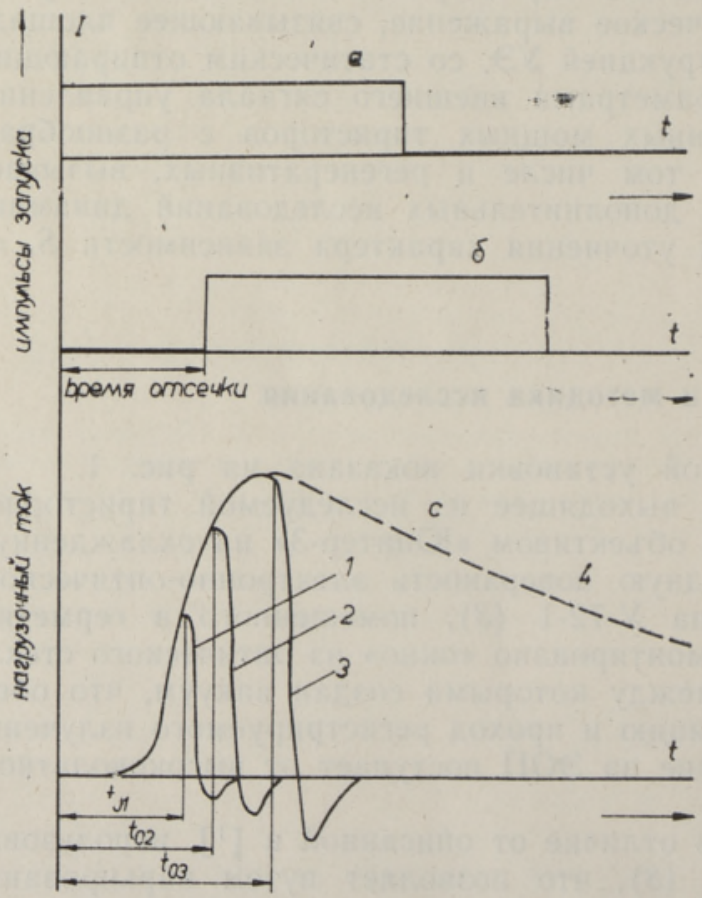

Рис. 2. Іиринцип функционировання аппаратуры по исследованию динамики образования НОВ: $a$ - импульс запуска тиристора, б - импульс запуска блока отсечки, $c$ - нагрузочные токи тиристора (1-3) и RC-контура (4).

РФ-3 и величина ее измеряется методом взвешивания засвеченных частей фотобумаги.

Принцип функционирования аппаратуры при включении тиристора показан на рис. 2. 


\section{Результаты исследований}

Экспериментальные образцы представляли собой $p-n-p-n$-структуры с различными по конфигурации и длине $(L)$ УЭ. По электрофизическим параметрам и технологии изготовления они были аналогичны выпускаемым промышленностью тиристорам серии ТЧ.

Регистрация рекомбинационного инфракрасного излучения (РИКИ) на исследуемых структурах в процессе включения показана на рис. 3 .
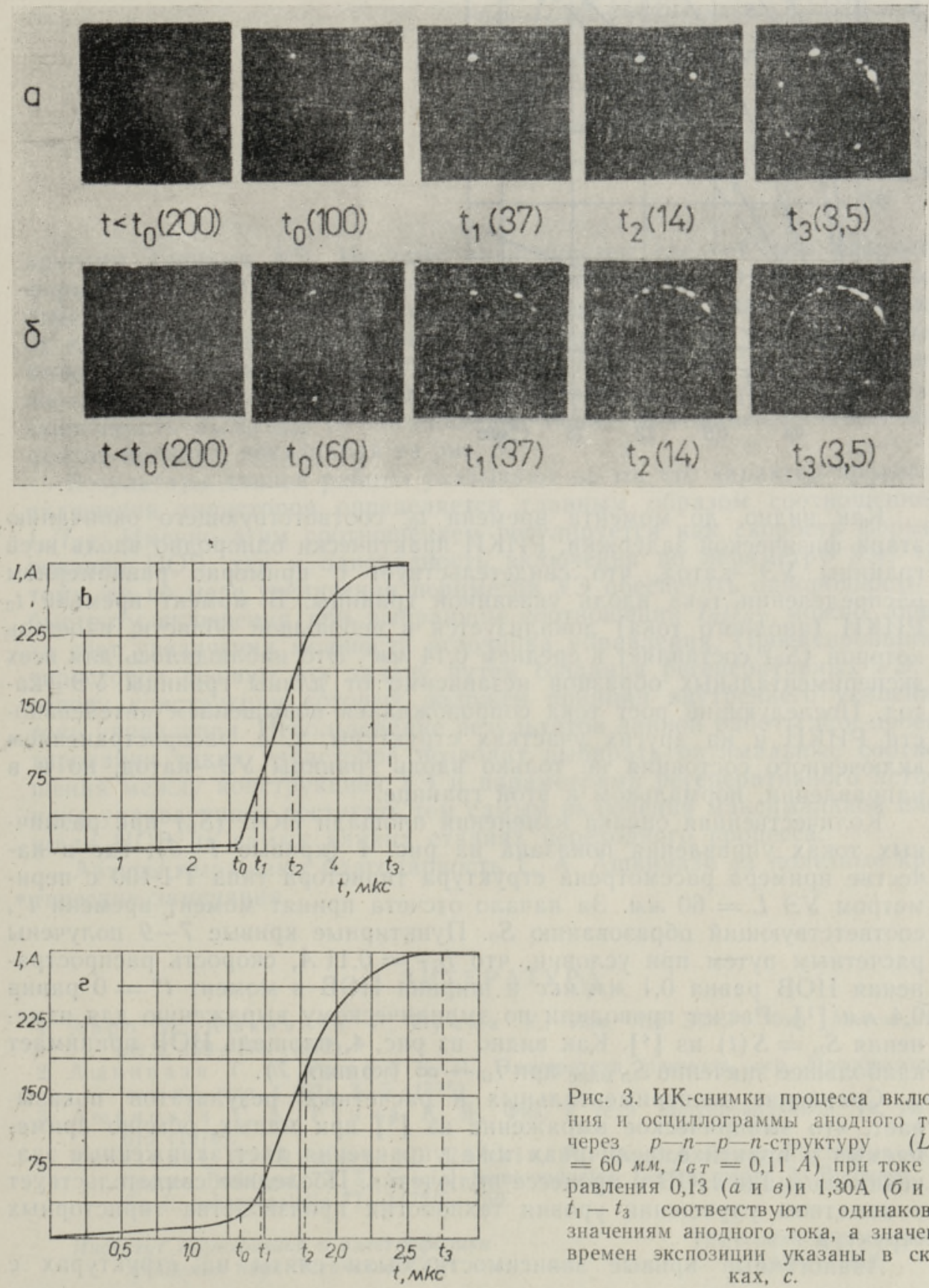

Рнс. 3. ИК-снимки процесса включения н осциллограммы анодного тока через $p-n-p-n$-структуру $(L=$ $=60 \mathrm{\mu м}, I_{G T}=0,11$ A) при токе управления 0,13 ( $a$ и $в$ ) и 1,30A (б и 2$)$; $t_{1}-t_{3}$ соответствуют одинаковым значениям анодного тока, а значения времен экспозицин указаны в скобках, $c$. 


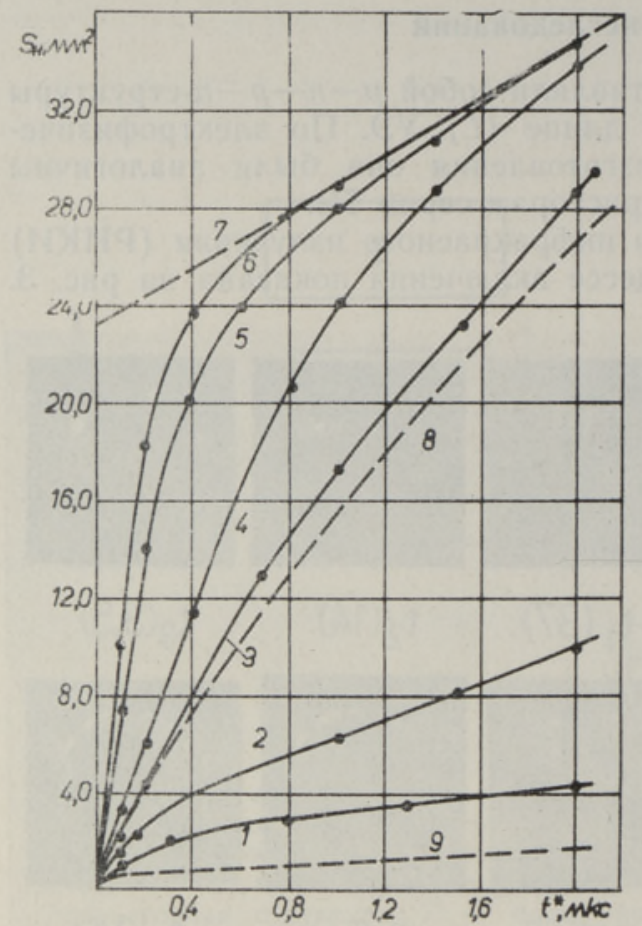

Рис. 4. Зависимость изменения площади НОВ в процессе нарастания анодного тока через $p-n-p$-n-струкrypy $\left(L=60 \mathrm{M \mu}, I_{G T}=0,11 A\right)$ от амплитуды тока управления: $I_{G}=0,13$ (l) $, 0,15$ (2), 0,25 (3 и 9), 1,0 (4), 3,0 $\left(\begin{array}{lllll}5 & \text { и } & 8\end{array}\right), 6,0 A \quad(6) ; I_{G} \rightarrow \infty \quad(7)$.

Как видно, до момента времени $t_{0}$, соответствующего окончанию этапа физической задержки, РИКИ практически однородно вдоль всей границы УЭ-катод, что свидетельствует о примерно равномерном распределении тока вдоль указанной границы. В момент времени $t_{0}$ РИКИ (анодного тока) локализуется в небольшой области, площадь которой $\left(S_{0}\right)$ составляет в среднем $0,14 м \mu^{2}$. Это наблюдалось для всех экспериментальных образцов независимо от длины границы УЭ-катод. Последующий рост тока сопровождался повышением интенсивности РИКИ и на других участках структуры, т. е. распространением включенного состояния не только вдоль границы УЭ-катод, но и в направлении, нормальном к этой границе.

Қоличественная оценка изменения площади $\mathrm{HOB}\left(S_{\text {п }}\right)$ при различных токах управления показана на рис. 4 (кривые $1-6$ ), где в качестве примера рассмотрена структура тиристора типа ТЧ-100 с периметром УЭ $L=60$ м.. За начало отсчета принят момент времени $t^{*}$, соответствующий образованию $S_{0}$. Пунктирные кривые $7-9$ получены расчетным путем при условии, что $I_{G T}=0,11 \mathrm{~A}$, скорость распространения НОВ равна 0,1 м.⿲/лкс и ширина НОВ в момент $t^{*}=0$ равна 0,4 мм [ $\left.{ }^{1}\right]$. Расчет проводили по эмпирическому выражению для изменения $S_{\mathrm{H}}=S(t)$ из $\left[{ }^{4}\right]$. Как видно из рис. 4 , площадь НОВ принимает

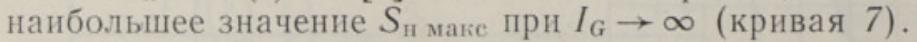

Сравнение экспериментальных и расчетных результатов показывает, что эмпирическое выражение из [ $\left.{ }^{4}\right]$ при малых, обычно применяемых в практике, величинах тока управления дает заниженные значения площади НОВ в процессе включения. Последнее свидетельствует о заметном улучшении уровня технологии производства тиристорных структур.

Аналогичные кривые завнсимости были сняты на структурах с 
Рис. 5. Зависимость изменения площади НОВ в процессе нарастания анодного тока через $p-n-p$-n-структуру от соотношения $I_{G} / I_{G T}$ при $t^{*}=0,1 \quad(1)$, 0,5 (2), 1 (3), и 2 sкк (4).

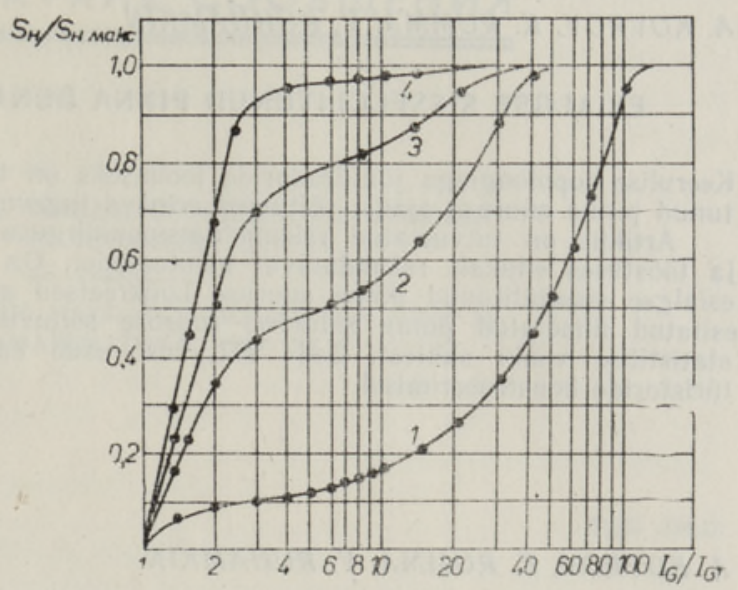

другими длинами УЭ. Из этих зависимостей следует, что скорость формирования НОВ определяется током управления. Однако, как уже отмечалось в $\left[{ }^{4}\right]$, характер изменения площади НОВ зависит не только от параметров внешнего сигнала управления, но и от значения статического отпирающего тока управления $\left(I_{G T}\right)$. А так как последний определяется периметром $У Э$, то, естественно, представляет интерес зависимость $S_{\mathrm{H}} / S_{\text {н маке от }}$ соотношения $I_{G} / I_{G T}$ для фиксированных моментов времени, показанная на рис. 5 .

Результаты данной работы указывают на то, что характер процесса включения тиристоров определяется главным образом соотношением $I_{G} / I_{G T}$. Именно этим соотношением регулируется как скорость фермирования НОВ, так и однородность включения по периметру УЭ. К тому же по мере увеличения периметра УЭ обеспечить однородность и быстроту включения при выбранном соотношении $I_{G} / I_{G T}$ можно лишь за счет увеличения мощности источника управления. Проектировщики тиристоров решают эту задачу путем создания регенеративных УЭ, в основу конструкции которых положен принцип внутреннего усиления тока управления. Использование результатов данной работы при проектировании таких тиристоров позволит выбирать оптимальные соотношения между конструкцией УЭ и параметрами регенеративного источника управления и достигать тем самым включения максимальной площади НОB на самых ранних стадиях переходного процесса.

Авторы выражают благодарность Г. А. Ашкинази за полезные критические замечания.

\section{Л ИТЕРАТУ РА}

1. Арро И., Ашкинази Г., Румма К., Изв. АН ЭССР, Физ. Матем., 21, № 2, 169-173 (1972).

2. Ашкин ази Г. А., Р умм а К. Я., Электротехн. пром-сть, сер. Преобразоват. техника, вып. 4 (63), 3-5 (1975).

3. Ашкинази Г. А., Румм а К. Я., Физ. и техн. полупроводников, 10, № 3 , 615 (1976).

4. Ashkinazi, G., Rumma, K., Rabkin, P., In: 3rd Power Electronics Conference Budapest 77. 5. Special Semiconductor Devies, paper 3, Budapest, $M$. Sz. E. sokszorosítója $77-1472 / 5-150$. 


\section{A. KOVROV, K. RUMMA, V. RUHHAMKIN}

\section{ESIALGSE SISSELULITUNUD PINNA DUNAAMIKA JOUTURISTORIDES}

Keerulise topoloogiaga jõutüristoride loomiseks on tarvilik täpsustada esialgse sisselülitunud pinna suuruse ajalisi sõltuvusi erineva tugevusega juhtvoolu korral.

Artiklis on tutvustatud rekombinatsioonkiirguse registreerimise pōhimôttel töötavat ja tööstuses edukalt rakendatavat mõôteseadet. On toodud ringelektroodiga türistoride esialgse sisselülitunud pinna suuruse konkreetsed ajalised sōltuvused. Esmakordselt on esitatud nimetatud pinna suhtelise suuruse sõltuvus juhtvoolu ja avamiseks tarviliku statistilise voolu suhtest. Seda sõltuvust saab edukalt kasutada võimendusega jōutüristoride konstrueerimisel.

\section{A. KOVROV, K. RUMMA, V. RUHAMKIN}

\section{FORMATION DYNAMICS OF INITIAL TURN-ON REGION IN POWER THYRISTORS}

Amplifying gates of complicated topology and with longer perimeters than the previous ones are used in designing power thyristors. The efficiency of such thyristors in pulse and high frequency modes is essentially determined by the initial turn-on region (ITR). Therefore a more detailed specification of the ITR value time dependence at different values of the gate direct current is necessary. In the present paper an apparatus for industrial use, based on registration of the infrared recombination radiation, is introduced. The necessary time resolution is obtained by interruption of the anode current. Results of the experiment show that the initial turn-on (independent of the amplitude of the gate direct current) begins at a single point and spreads along the perimeter with the speed related to the amplitude of the gate direct current. The corresponding data are compared with the empirical formula of the ITR area presented earlier. A relationship between the relative change of the ITR area and the ratio of the gate direct current to the minimum value of this current is presented. The established relationship is well applicable to designing of amplifying power thyristors and in determining the gate direct current necessary for efficient iunctioning of power thyristors. 